\title{
FACTOR DE DESCUENTO, FLUJOS FUTUROS Y DETERIORO DE ACTIVOS TANGIBLES E INTANGIBLES
}

\section{DISCOUNT FACTOR, FUTURE FLOWS AND DETERIORATION OF TANGIBLE AND INTANGIBLE ASSETS}

RaúlAiberto Arrarie Mera*

Docente Principal de la Facultad de Ciencias Contables

Universidad Nacional Mayor de San Marcos-UNMSM / Lima-Perú

[Recepción: agosto 2016/ Conformidad: Setiembre 2016]

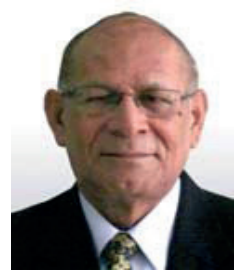

\section{RESUMEN}

Este trabajo, es un análisis exploratorio-descriptivo-cuantitativo; simulación matemática de un flujo creciente y decreciente de ingresos futuros a tasas bajas y altas de interés; para deducir sus resultados. Objetivos:

a) Evaluar un factor de descuento justo y razonable para calcular la proyección de los flujos futuros de los bienes de activos fijos tangibles e intangibles.

b) Verificar cómo estimar el valor recuperable de una Unidad Generadora de Efectivo (UGE).

c) Estudiar en qué medida normar una tasa de interés global es conveniente a la lucha contra el lavado de activos y la corrupción.

Concluimos: si aplicamos una tasa de interés elevada afectamos a la empresa con menos utilidades; $y$ al Estado con menores ingresos tributarios. Si la tasa es baja, sucede lo contrario. Confirmamos que es conveniente para nuestros países la normalización trimestral de las tasas de interés a nivel global por parte del IASB.

\section{Palabras Clave:}

Deterioro de Activos Fijos, Factor de Descuento, Flujos futuros, Tasas de Interés, Valor Futuro, Valor de Uso.

\begin{abstract}
This work is an exploratory-descriptive-quantitative analysis; a mathematical simulation of an increasing and decreasing flow of future income at low and high interest rates; to deduce their results. Goals:

A) To evaluate a fair and reasonable discount factor to calculate the projection of future flows of tangible and intangible fixed assets.

B) To verify a way to estimate the recoverable value of a Cash-Generating Unit (CGU).

C) To study the extent to which a global interest rate is appropriate to fight against money laundering and corruption.

We conclude: if we apply a high interest rate we affect the company with less profits and the State with lower tax revenues. If the rate is low, the opposite is true. We confirm that it is convenient for our countries to make quarterly normalization of interest rates at the global level by the IASB.
\end{abstract}

\section{Keywords:}

Impairment of Fixed Assets, Discount Factor, Future flows, Interest rates, Future Value, Use Value.

\footnotetext{
* Doctor en Ciencias Contables y Empresariales y Contador Público - UNMSM. Email: rarrartem@unmsm.edu.pe
} 


\section{INTRODUCCIÓN}

\section{Planteamiento del Problema.}

¿Por qué se ha realizado esta investigación? Por el interés que despierta en el contexto científico contable la discusión técnica del deterioro del valor de los Activos Fijos Tangibles e Intangibles promovidos por la Norma Internacional de Contabilidad $\mathrm{N}^{\circ} 36$, (NIC), que emite la Junta de Estándares Internacionales de Contabilidad (IASB o International Accounting Standards Board) y examinar de paso el cambio de paradigma que acontece en el análisis económico empresarial. ¿Quién fija el factor de descuento o costo de capital, para calcular la proyección de los flujos futuros del valor de uso de un activo fijo? ¿Por qué el IASB no publica trimestralmente una tasa de interés normalizada a nivel mundial, segmentada por continentes para efectos del cumplimiento de la NIC 36? Así se zanjaría el problema de valuación aplicando una tasa de costo de capital internacional, debidamente normada. Permitiendo repartir entre los accionistas, utilidades efectivamente generadas y presentar estados financieros confiables consecuentes con la economía real y no imaginaria. Evitando a futuro el abuso del valor razonable y su descrédito consiguiente.

Un interesante artículo publicado en Lima fechado el 09/09/2016, firmado por Martínez, J (2016: 21), decía, entre otras cosas importantes, según datos del Banco Central Europeo que "a inicios del siglo XXI una persona que depositaba su dinero en un banco habría tenido que esperar 18 años para ver que estos fondos se duplicaban”. Es decir los bancos pagaban $5.56 \%$ promedio anual. "Bajo las condiciones financieras actuales, esa persona ahora tendría que esperar 122 años”. En otras palabras nos está diciendo que los bancos han reducido su tasa a $0.82 \%$ promedio anual. Problema que no sólo ocurre en Europa. Sucede en Japón y Suiza, donde las tasas de interés son cercanas a cero. "Y es muy probable que ese fenómeno se prolongue por mucho tiempo". ¿Qué ha generado esta situación? El articulista nos explica por "los cambios tecnológicos, el envejecimiento de la población, la liberalización del comercio y de los flujos de capital se han combinado para reducir el nivel de crecimiento económico y crear presiones deflacionarias en muchas partes del mundo". Así de claro, el problema está planteado. Si la tasa de interés disminuye beneficia al factor de descuento, lo que hará que aumente de manera significativa el valor recuperable.

\section{Justificación.}

La disyuntiva que plantea la NIC 36, está en establecer el mayor valor recuperable en el valor neto de realización (precio de venta neto) y el valor de utilización económica (valor de uso). Porque uno solo de ellos, superior al valor en libros, nos permite evitar el cálculo. Pues el valor en uso representa el valor presente de los futuros flujos de caja que la entidad espera obtener a través del uso del activo y, en su caso, de su venta al finalizar la vida útil del mismo.

Como es de notar el tema ha generado discusiones en todos los ámbitos económico-financieros del Perú y del mundo, y en particular a nivel de los Contadores Públicos en calidad de auditores independientes que por ley tienen la delicada función de dar opinión sobre la situación de los estados financieros que se publican en las Bolsas de Valores en forma trimestral, y en los países del globo a fin de año, para efectos del cálculo del impuesto a la renta. Si aplicamos una tasa de descuento menor afectamos los resultados de la empresa con pérdida por deterioro y con mínimas utilidades; y a los ingresos del Estado con menores impuestos. Por el contrario, si la tasa de descuento es elevada, ocurre todo lo opuesto. No existe deterioro y todos los grupos de interés felices. Consiguientemente, para obviar esta discusión lo ideal está en el "justo medio".

\section{MARCO TEÓRICO}

\section{Antecedentes}

Revisando trabajos de investigación y tesis en Repositorios Universitarios de la República Peruana, se han encontrado algunos trabajos con enfoques al deterioro de activos tangibles e intangibles (Ver Tabla $\mathrm{N}^{\circ} 1$ ). 
Tabla No 01

Resumen de Evaluaciones

\begin{tabular}{|c|c|c|c|c|c|c|c|}
\hline \multirow[t]{2}{*}{ Indicadores } & \multicolumn{7}{|c|}{$\begin{array}{c}\text { Distribuidoras } \\
\text { Unidades Generadoras de Efectivo }\end{array}$} \\
\hline & A & B & $\mathrm{C}$ & $\mathrm{D}$ & $\mathrm{E}$ & $\mathrm{F}$ & G \\
\hline \multicolumn{8}{|l|}{ Externos } \\
\hline \multicolumn{8}{|l|}{ Cambios adversos que ocurrieron o se espera que ocurran en el futuro cercano en: } \\
\hline Ambiente Tecnológico & No & No & No & No & No & No & No \\
\hline Ambiente Legal & No & No & No & No & No & No & No \\
\hline Ambiente del Mercado & No & No & No & No & No & No & No \\
\hline Aumento de Tasas de interés en el mercado que pudiera causar algún impacto & No & No & No & No & No & No & No \\
\hline Desperfectos, cancelación de productos & No & No & No & No & No & No & No \\
\hline $\begin{array}{l}\text { El valor en libros de los activos netos es mayor que la capitalización del mer- } \\
\text { cado }\end{array}$ & No & No & No & No & No & No & No \\
\hline \multicolumn{8}{|l|}{ Internos } \\
\hline Deterioro de los flujos de efectivo en comparación con los presupuestos & No & No & No & No & No & No & No \\
\hline $\begin{array}{l}\text { Deterioro de los flujos de efectivo presupuestados en la ganancia o pérdida } \\
\text { operativa, por separado o en combinación con las cifras de periodo actual. }\end{array}$ & No & No & No & No & No & No & No \\
\hline Evidencia de obsolescencia o daño físico. & No & No & No & No & No & No & $\mathrm{Si}$ \\
\hline $\begin{array}{l}\text { Ocurrieron cambios adversos significativos o se espera que ocurran en el futu- } \\
\text { ro cercano, acerca de la manera en que se usan los activos }\end{array}$ & No & No & No & No & No & No & No \\
\hline $\begin{array}{l}\text { Se incluyen planes de reestructuración y planes para retirar el activo antes de } \\
\text { lo esperado. }\end{array}$ & No & No & No & No & No & No & $\mathrm{Si}$ \\
\hline
\end{tabular}

Fuente: Gallado, E. (2010). Evaluación del Deterioro del Valor de los Activos Fijos en una Empresa dedicada a la fabricación de Helados. Tesis para Contador Público y Auditor. Universidad de San Carlos de Guatemala.

Gallardo, E (2010: 76-106). Este trabajo de investigación se ubica en el Municipio de San Lucas, departamento de Sacatepéquez, en la República de Guatemala. Esta referida a una empresa dedicada a la producción y distribución de helados en el mercado nacional y Centro América.

Al31 de diciembre del 2007, la empresa presentaba en su estado de situación 69 millones de quetzales en activos fijos sobre un total de 93 millones en moneda nacional. Es decir, el 74\% de su inversión total. $Y$ las utilidades antes de impuestos que generaba la empresa en ese mismo periodo, era de $16 \%$ sobre el patrimonio.

En el aspecto de indicadores externos se analizaron: tasas de interés aplicables, evaluación de clientes y proveedores principales, valuación de activos; y no se identificaron cambios importantes. (Ver Tabla $\mathrm{N}^{\circ} 01$ ).
En cuanto a indicadores externos se evaluaron presupuestos planteados a finales de diciembre 2006 con respecto a las cifras obtenidas en el 2007.

La pérdida por deterioro del activo, es la cantidad en que excede el valor en libros del conjunto de la unidad generadora de efectivo, de su importe recuperable, que en este caso se determinó por medio del valor de uso. El efecto de este ajuste se refleja en el resultado del periodo actual. No hay una explicación puntual para haber establecido la tasa de descuento en $6 \%$. (Ver Tabla N 02)

Sumba, D. \& Sigua, M. (2012: 15-39). El objetivo de este trabajo consiste en establecer los procedimientos que deben ser aplicados para asegurarse que los activos de la Compañía de Transportes Arcentales Cía. Ltda.,(Cuenca, Ecuador), estén contabilizados por un importe que no sea superior a su importe recuperable. En situaciones extremas la tasa puede estar representada por el costo del capital ponderado de 


\section{Tabla No 02}

Distribuidora G (Petén). Valor presente de los Flujos de Efectivo Esperados (cifras expresadas en Quetzales)

\begin{tabular}{|c|c|c|c|}
\hline AÑOS & $\begin{array}{c}\text { INGRESOS } \\
\text { PROYECTADOS }\end{array}$ & $\begin{array}{c}\text { FACTOR } \\
\text { DE DESCUENTO }\end{array}$ & VALOR PRESENTE A 2007 \\
\hline 2008 & $8,177,222$ & $6 \%$ & $7,714,360$ \\
\hline 2009 & $5,724,055$ & 0.9433962 & $5,094,389$ \\
\hline 2010 & $4,293,042$ & 0.8899964 & $3.604,520$ \\
\hline 2011 & $3,434,433$ & 0.8396193 & $2,720,393$ \\
\hline \multirow[t]{2}{*}{2012} & $3,221,772$ & 0.7920937 & $2,407,496$ \\
\hline & $24,850,524$ & & $21,541,158$ \\
\hline \multicolumn{3}{|c|}{ Valor en Libros al 31 de diciembre de 2007} & $27,029,384$ \\
\hline \multicolumn{3}{|l|}{ Deterioro } & $(5,488,226)$ \\
\hline
\end{tabular}

Fuente: Gallardo E. (2010).

una entidad. Representa un problema cuya solución depende del uso del juicio profesional y la capacidad que la administración de una entidad tenga para el establecimiento de dicha tasa de descuento y de las bases, suposiciones e hipótesis que le dan sustento.

En el caso práctico que presentan los autores consideran como tasa de descuento la tasa de interés activa asociada con el préstamo obtenido por el accionista y que sirvió para la adquisición del vehículo. Bajo las circunstancias del caso, según ellos, esta es la tasa que refleja mejor los riesgos y beneficios inherentes al funcionamiento del vehículo (10\%). Tasa que sirvió para calcular el VAN. (Valor Actual Neto).

\section{Valor Razonable según la NIC No. 36.}

\section{Según Ferrer, A. (2010: IV-6).}

"Para la determinación de este valor razonable se requiere que la gerencia fundamente sus proyecciones de flujos de efectivo ya que éstos resultan importes claves en el cálculo, también lo es la identificación de la tasa de descuento”.

Analicemos el caso práctico de Ferrer, A. (2013: IV-13), nos ha llamado la atención por la tasa de descuento que se utiliza: $15 \%$. Lo desarrollaremos in-extenso, por ser un tema que en el mediano plazo se dará en el Perú: por cambio de tecnología.

\section{Desvalorización de Planta Industrial Enunciado}

La empresa Concentrados Eléctricos construyó una planta eléctrica a base de petróleo que ha estado usando por varios años, siendo su valor neto en libros al 31 de diciembre del 2010 de S/.800, 000.

Debido al alza del precio del petróleo, la compañía ha comprado una nueva planta eléctrica a base de gas natural. En los próximos años, solo va a utilizar la planta a base de petróleo cuando exceda la capacidad de la nueva planta y por consiguiente, su flujo de efectivo va a disminuir.

La empresa ha dispuesto efectuar un estudio de desvalorización de la mencionada planta. ¿Cómo determinar la desvalorización de la planta eléctrica?

1. Planteamiento del Problema.

a) Determinar el valor razonable (valor de venta).

b) Establecer el valor de uso:

- Definir el flujo de efectivo

- Identificar la Tasa de Interés para calcular el factor de descuento.

c) Estimación de la desvalorización.

d) Registro contable.

2. Desarrollo del Caso

a) Determinar el valor razonable (valor de venta)

Por tasación de Perito Independiente =

$\begin{array}{lll}\text { Valor razonable } & \text { S/. } & 700,000 \\ \text { Gastos de desmontaje } & \text { S/. } & (100,000) \\ \text { Valor de Venta } & \text { S/. } & 600,000\end{array}$


b) Establecer el valor de uso

Tasa de interés, según condiciones actual del mercado $=15 \%$

Factor de descuento $=1 /(1+\mathrm{i})^{\mathrm{n}}$

F. D. = Factor de Descuento =?

i $\quad=$ tasa de interés $=15 \%$ anual

$\mathrm{n}=$ número de periodos $=8$ años

Proyección de flujo de efectivo para los próximos 8 años que le queda de vida útil a la planta. (Ver Tabla $\mathrm{N}^{\circ} 3$ )

3. Estimación de la desvalorización.

Valor Razonable (Venta)

$$
\text { S/. } \quad 600,000
$$

Valor de Uso (Ver Tabla Nº 3).

$$
\text { S/. } \quad 503,090
$$

Se elige el valor razonable por ser el mayor. Luego se confronta con el valor en libros:

\begin{tabular}{lll} 
Valor en libros & S/. & $\begin{array}{l}800,000 \\
\text { Valor Razonable (Venta) }\end{array}$ \\
S/. & $(600,000)$ \\
\cline { 2 - 2 } Monto a Desvalorizar & S/. & $\begin{array}{l}200,000 \\
=====\end{array}$
\end{tabular}

4. Registro Contable.

68 VALUACIÓN Y DETERIORO DE ACTIVOS Y PROVISIONES 200,000

685 Deterioro del valor de los activos

36 DESVALOR IZACIÓN DE ACTIVO

INMOVILIZ.

200,000

3632 Instalaciones

$\mathrm{x} /$ Provisión del gasto.

94 GASTOS DE ADMINISTRACION 200,000

79 CARGAS IMPUTABLES A CUENTAS

DE COSTOS

200,000

$\mathrm{x} /$ Destino del gasto

En resumen, notamos aquí, de tres trabajos desarrollados a nivel de América Latina, el siguiente resultado en cuanto a tasas de interés aplicado a flujos futuros: Ecuador 10\%. Guatemala 6\%. Perú $15 \%$. Son diferentes realidades, pero un solo problema. Es decir, buscamos la unidad en la diversidad.

Tabla No 03:

Empresa Concentrados Eléctricos. Flujos Futuros de Efectivo Descontado (valor expresado en miles de soles)

\begin{tabular}{|c|c|c|c|}
\hline AÑOS & $\begin{array}{c}\text { INGRESOS } \\
\text { PROYECTADOS }\end{array}$ & $\begin{array}{c}\text { FACTOR DE } \\
\text { DESCUENTO } \\
15 \%\end{array}$ & $\begin{array}{c}\text { FLUJO DE } \\
\text { EFECTIVO } \\
\text { DESCONTADO }\end{array}$ \\
\hline 2011 & 180 & 0.8695652 & 156.52 \\
\hline 2012 & 150 & 0.7561437 & 113.42 \\
\hline 2013 & 120 & 0.6575162 & 78.90 \\
\hline 2014 & 100 & 0.5717532 & 57,18 \\
\hline 2015 & 85 & 0.4971767 & 42.26 \\
\hline 2016 & 60 & 0.4323276 & 25.94 \\
\hline 2017 & 42 & 0.3759370 & 15.79 \\
\hline 2018 & 40 & 0.3269018 & 13.08 \\
\hline TOTAL & 777 & VALOR DE USO & 503.09 \\
\hline
\end{tabular}

Fuente: Ferrer, A. (2013, IV-11) Revisando las NIIF: NIC 36 Deterioro del Valor de los Activos. Revista "Actualidad Empresarial" No. 277. Segunda Quincena de Abril 2013. Lima. Perú. 


\section{Bases Teóricas Científicas}

\section{NIC 36. Deterioro de Activos Fijos}

\section{Resumen de la Norma.}

\section{Objetivo.}

- Establecer los procedimientos que se deben aplicar para asegurar que los activos están contabilizados por un importe que no sea superior a su importe recuperable.

- Reconocer una pérdida por deterioro del valor del activo que esté sobreestimado.

- Cuándo se revertirá la pérdida por deterioro del valor.

- La información a revelar.

\section{Alcance.}

Aplicable a los activos o Unidad Generadora de Efectivo, (UGE), diferentes a los detallados en la Tabla Nº4.

\section{Indicación de un Activo que podría estar deteriorado}

- Fuentes Externas de Información:

a) El valor de mercado del activo ha disminuido significativamente más de lo que cabría esperar como consecuencia del paso del tiempo o de su uso normal.

b) Cambios significativos con una incidencia adver- sa, referentes al entorno en que operar, o bien en el mercado al que está destinado el activo.

c) Las tasas de interés del mercado han sufrido incrementos que probablemente afecten a la tasa de descuento utilizado para calcular el valor en uso del activo.

d) El importe en libros de los activos netos, es mayor que su capitalización bursátil.

- Fuentes Internas de Información:

a) Se dispone de evidencia sobre la obsolescencia o deterioro físico de un activo.

b) Han tenido lugar, o se espera que tengan lugar cambios significativos en el alcance o manera en que se usa o espera usar el activo, que afectarán desfavorablemente a la entidad.

c) Se dispone de evidencia procedente de informes internos, que indica que el rendimiento económico del activo es, o va ser, peor que el esperado.

\section{Tasa de Interés, Factor de Descuento y Flujos de Caja a futuro}

Económicamente hablando, la tasa de interés es el precio del dinero en el mercado financiero o el porcentaje que paga un capital invertido en un período de tiempo. La tasa de interés es fijada por el banco central de cada país a los otros bancos que, a su

Tabla No 04

Activos no competencia de la NIC. no. 36

\begin{tabular}{|l|c|}
\hline \multicolumn{1}{|c|}{ CUENTAS DE ACTIVO } & COMPETENCIA DE \\
\hline Existencias & NIC No. 2 \\
\hline Contratos de Construcción & NIC No. 11 \\
\hline Activos Tributarios Diferidos & NIC No. 12 \\
\hline Activos procedentes de retribuciones a los empleados & NIC No. 19 \\
\hline Activos Financieros (dinero o efectivo; cuentas por cobrar, valores) & NIC No. 39 \\
\hline Activos Inmobiliarios (medidos a valor razonable) & NIC No. 40 \\
\hline Activos Biológicos (medidos a valor razonable) & NIC No. 41 \\
\hline $\begin{array}{l}\text { Costos de adquisición diferidos, así como activos intangibles derivados de } \\
\text { los derechos contractuales de una aseguradora en contratos de seguros }\end{array}$ & NIFF No. 04 \\
\hline Activos nos corrientes clasificados como mantenidos para la venta. & NIIF No. 05 \\
\hline
\end{tabular}

Fuente: Fundación del Comité de Estándares Internacionales de Contabilidad. (International Accounting Standards Committee Foundation. IASCF). Londres. Gran Bretaña. 
vez, la fijan a los sujetos de crédito por los préstamos otorgados.

Según las matemáticas financieras, los factores de descuento se utilizan para calcular el valor actual de cualquier flujo de caja. Sin embargo, antes de continuar con el desarrollo de estas ideas, es necesario precisarconceptos sobre qué es una tasa de interés desde el punto de vista de las normas internacionales de información financiera. No es igual decir costo de capital y costo de oportunidad. El primero está referido al costo del dinero en el mercado, al capital ajeno, a los préstamos bancarios. Mientras que el segundo, es la tasa de rentabilidad que desea el empresario, o también denominado "costo de oportunidad", proveniente del capital propio. Por tanto, al referirnos al factor descuento en el caso particular de calcular el deterioro de los activos tangibles e intangibles, nos estamos refiriendo al costo del dinero en el mercado, a las tasas de interés que cobran o fijan los bancos comerciales. Por tanto, la tasa de interés que establece un banco tiene dos componentes incluidos: el tipo de interés libre de riesgo + compensación por riesgo. No es correcto agregar a esta tasa un nuevo factor de riesgo, porque la tasa de mercado ya tiene incluido su factor de riesgo. Tampoco es correcto utilizar el modelo del costo del capital promedio ponderado conocido como WACC (Weighted average cost of capital), porque representa el promedio de todas las fuentes de fondos, (tanto capital ajeno como propio), identificado en un momento determinado en el tiempo de la empresa en marcha.

\section{Tasas de interés del mercado internacional}

Según Cruz, S. (2000: 1-5), pone de manifiesto las distintas alternativas con las que se encuentra el evaluador de una empresa a la hora de estimar el tipo de actualización o descuento necesario para determinar el valor de la empresa en base a sus rentas futuras estimadas (beneficios o cash-flows), esto es, para calcular el valor de rendimiento, pueden ser elegidos entre las siguientes:

a) Tipo base que su vez los clasifica en tres categorías:

1) Tipos básicos de interés,
2) Tipos de los mercados nacionales, $y$

3) Tipos de mercado internacionales.

b) Prima de riesgo, $y$

c) Efecto de la inflación.

Analizaremos a continuación las tasas de interés del punto a), por ser temas que interesan a nuestro análisis:

1) El Prime rate norteamericano.

2) LIBOR

\section{El Prime Rate norteamericano}

Posterior a la "Gran Depresión”, que se conoce en la historia, nace en los EE.UU de América en el año 1933. Se produjo una importante caída de la demanda de crédito en los Estados Unidos, lo que provocó una fuerte competencia entre los bancos para captar los pocos clientes de bajo riesgo que todavía quedaban, con la consiguiente caída de los tipos de interés. Ello determinó que los bancos tuvieran que ponerse de acuerdo en la denominada Prime Rate Convention para regular el mercado de capitales en EE.UU. de N. A. y en el mundo ya que la mayoría de operaciones financieras mundiales se realizan en dólares. Como es una tasa que todo el mundo conoce, permite que sea la tasa de referencia a nivel mundial y generen en el mercado financiero: confianza, tranquilidad, seguridad y crecimiento.

Según Paredes, M (2012: 3), de la Superintendencia de Bancos y Seguros del Ecuador:

"La tasa preferencial de préstamos Prime Rate es aquella que los bancos estadounidenses cobran a sus mejores clientes sobre sus préstamos. Constituye una referencia del nivel de las tasas activas de ese país. Obviamente, por muy buenos clientes que sean, esta tasa siempre será un poco más alta que la tasa de descuento que le cobra la FED al banco directamente. En los mercados internacionales de dinero siempre son muy consideradas las tasas de interés de los depósitos a la vista y a plazos dentro de cada país”.

"La tasa prime constituye una tasa mundial que varía constantemente en función de la demanda del crédito y de la oferta monetaria; pero también en función de las expectativas inflacionarias y los resultados de la cuenta corriente en la balanza de pagos de los Estados Unidos de América”. 


\section{La Tasa LIBOR}

Según Zanabria, P. (2009: 49-51), LIBOR, del acróstico en inglés London Inter Bank Offered Rate, desde 1984 a la fecha, la Asociación de Bancos Británicos "empezó a producir una encuesta de las tasas de préstamos interbancarios en Londres ... la cual ha venido siendo usada en forma creciente como una tasa de interés global".

Para Kiff, J. (2012: 31/33), experto principal del sector financiero del FMI, la tasa LIBOR es importante "debido a su uso generalizado como referencia para muchas otras tasas de interés a las cuáles efectivamente se opera". Según informes procedentes del Tesoro del Reino Unido, contratos financieros por más de US $\$ 300$ billones de dólares están ligados a la tasa LIBOR "y ese monto no incluye decenas de miles de millones de dólares en hipotecas para la vivienda a tasa ajustable y otros préstamos para consumo en todo el mundo que, de una manera $u$ otra, hacen referencia a la tasa LIBOR". "Siendo el dólar de EE.UU. la principal moneda a nivel mundial, las tasas LIBOR para depósitos en dólares son probablemente las más ampliamente utilizadas y citadas".

Sin embargo, como todas las cosas importantes, hay la controversia acerca de la forma cómo algunos bancos ingleses comunican las tasas a las cuales ellos "creen" que pueden tomar préstamos. Situación que ha venido ocurriendo desde 1986, inicialmente a solo tres monedas: el dólar, el yen y la libra esterlina. Hasta que en el 2012, surgió la denuncia de manipular la tasa LIBOR, cargo que reconoció el gran banco británico Barclays "que en junio de 2012 aceptó pagar multas por aproximadamente US $\$ 450$ millones a los organismos reguladores del Reino Unido y de Estados Unidos. Monto que según comentarios de la prensa internacional de la época no representó ni siquiera el $5 \%$ de sus ganancias generadas.

\section{HIPÓTESIS}

\section{Hipótesis General}

La tasa de descuento para la proyección de flujos futuros, determina el valor recuperable en mayor o menor cuantía, del deterioro de los activos fijos tangibles e intangibles.

\section{Hipótesis Específicas}

1. La tasa de descuento para la proyección de flujos futuros, es factor determinante para establecer que no haya deterioro contable entre su valor razonable menos los costes necesarios para su venta y su valor en uso.

2. La tasa de descuento para la proyección de flujos futuros, normada por una tasa de interés global coadyuva a la lucha contra el lavado de activos y la corrupción.

\section{METODOLOGÍA}

Se utilizó una metodología no experimental de caso, fundamentalmente cuantitativa, exploratoria-descriptiva. La principal técnica que se manejó como recolección de información en este estudio fue el análisis económico-financiero. El instrumento utilizado fue construir un flujo creciente de ingresos futuros a tasas bajas y a tasas altas de interés; y/o un flujo decreciente de ingresos futuros a tasas bajas y a tasasaltas de interés, que permitieron identificar la situación actual a la problemática que se establece en la presente investigación. Es decir una aplicación matemática de simulación.

\section{RESULTADOS Y DISCUSIÓN}

\section{a. Análisis, Interpretación y Discusión de Re- sultados}

A continuación desarrollaremos una simulación matemática de dos flujos de caja ya mostrados en las Tablas $\mathrm{N}^{\circ} 2$ y 3 , a los que modificaremos las tasas de interés, lo que generará un nuevo factor de descuento. Simularemos con tasas al 6\% y $12 \%$. Y evaluaremos sus resultados. (Ver Tablas $\mathrm{N}^{\circ} 05$ y 06 )

\section{Comentario de la Tabla $\mathbf{N}^{\circ} 05$}

El análisis cuantitativo nos muestra que a mayor tasa de interés, (pasamos de 6\% a 12\%), el deterioro aumenta, por tanto, la empresa tiene mayor crédito fiscal, y pagará menos impuestos a la renta, afectando los intereses del Estado. 
Tabla No 05:

Distribuidora g (Petén). Valor presente de los Flujos de Efectivo Esperados (cifras expresadas en Quetzales)

\begin{tabular}{|l|l|l|c|c|c|}
\hline \multirow{2}{*}{ AÑOS } & \multirow{2}{*}{$\begin{array}{l}\text { INGRESOS } \\
\text { PROYECTADOS }\end{array}$} & \multicolumn{2}{|l|}{ FACTOR DE DESCUENTO } & \multicolumn{2}{l|}{ FACTOR DE DESCUENTO } \\
\cline { 3 - 6 } & $6 \%$ & VALOR PRESENTE & $12 \%$ & VALOR PRESENTE \\
\hline 2008 & $8,177,222$ & 0.9433962 & $7,714,360$ & 0.8928571 & $7^{\prime} 301,091$ \\
\hline 2009 & $5,7254,055$ & 0.8899964 & 5,094389 & 0.7971939 & $4^{\prime} 563,182$ \\
\hline 2010 & $4,293,042$ & 0.8396193 & $3,604,520$ & 0.7117802 & $3^{\prime} 055,702$ \\
\hline 2011 & $3,434,433$ & 0.7920937 & $2,720,393$ & 0.6355181 & $2^{\prime} 182,644$ \\
\hline 2012 & $3,221,772$ & 0.7472582 & $2,407,496$ & 0.5674269 & $1^{\prime} 828,120$ \\
\hline & & $21^{\prime} 541,158$ & & $18^{\prime} 930,739$ \\
\hline \multicolumn{2}{|l|}{ Valor en libros al 31 de diciembre 2007} & $27,029,384$ & & $27^{\prime} 029,384$ \\
\hline \multicolumn{2}{|l|}{ Deterioro } & $(5,488,226)$ & & $\left(8^{\prime} 098,645\right)$ \\
\hline
\end{tabular}

Fuente: Elaboración Propia.

Veamos a continuación la siguiente simulación matemática:

Tabla No 06:

Empresa Concentrados Eléctricos Flujos Futuros de Efectivo Descontado (valor expresado en miles de soles)

\begin{tabular}{|c|c|l|c|c|c|}
\hline \multirow{2}{*}{ AÑOS } & \multirow{2}{*}{$\begin{array}{l}\text { INGRESOS } \\
\text { PROYECTADOS }\end{array}$} & \multicolumn{2}{|c|}{ FACTOR DE DESCUENTO } & \multicolumn{2}{c|}{ FACTOR DE DESCUENTO } \\
\cline { 3 - 6 } & & $15 \%$ & VALOR PRESENTE & $7.5 \%$ & VALOR PRESENTE \\
\hline 2011 & 180 & 0.8695652 & 156.52 & 0.9302326 & 167.44 \\
\hline 2012 & 150 & 0.7561437 & 113.42 & 0.8653326 & 129.80 \\
\hline 2013 & 120 & 0.6575162 & 78.90 & 0.8049606 & 96.60 \\
\hline 2014 & 100 & 0.5717532 & 57.18 & 0.7488005 & 74.88 \\
\hline 2015 & 85 & 0.4971767 & 42.26 & 0.6965586 & 59.21 \\
\hline 2016 & 60 & 0.4323276 & 25.94 & 0.6479615 & 38.88 \\
\hline 2017 & 42 & 0.3759370 & 15.79 & 0.6027549 & 25.32 \\
\hline 2018 & 40 & 0.3269018 & 13.08 & 0.5607022 & 22.43 \\
\hline TOTAL & 777 & & 503.09 & & 614.56 \\
\hline Valor en Libros & & 800.00 & & 800.00 \\
\hline Deterioro & & $(296.91)$ & & $(214.56)$ \\
\hline
\end{tabular}

Fuente: Elaboración Propia. 


\section{b. Presentación de Resultados.}

A la luz de la información precedente, sustentada en términos cuantitativos, se deduce que la tasa de interés está en relación directa con el valor de deterioro. A mayor tasa de interés, más deterioro. Por tanto, es una variable que debe ser controlada. No puede dejarse al libre albedrío de los valuadores y/o los empresarios del mundo. Razón más que suficiente para controlarlo.

"¿Qué hubiera pasado si China esperaba hasta que la $\mathrm{FED}^{1}$ decidiera una subida de tasas para resolver, recién entonces, la devaluación del yuan renminbí?" se preguntaban Barbera, J \& Gutiérrez, A \& Siaba, J. (2015: 47). Concluyen estos economistas argentinos que "las decisiones económicas son partidas simultáneas que se juegan en un único tablero", el mundo. La coordinación es fundamental para ordenar el tráfico de este mundo global que nos tocó vivir.

\section{c. Nuestra Propuesta.}

Un método frecuentemente utilizado consiste en considerar el tipo de interés implícito de bonos gubernamentales al tipo de interés fijo o el tipo de interés interbancario, en el que los bancos se prestan dinero entre sí. Pero también existe el Prime Rate Norteamericano y la LIBOR del Reino Unido. El Euro de la Comunidad Europea, El Yen Japonés y el Yuan Chino.Consideramos perfectamente aceptable normar las tasas mundiales en base a estos parámetros más popularmente difundidas en el mundo. La iliquidez es un riesgo previsible y hay que evitarlo.

\section{CONCLUSIONES}

1. Actualmente, (noviembre 2016), las tasas de interés en el mundo desarrollado, "son motivo de preocupación pero no de alarma” según comentario del Presidente del Banco Central de Reserva del Perú. La Reserva Federal de EE. UU., de Norteamérica mantiene su tasa de interés de referencia en el rango de $0.25 \%$ a $0.5 \%$. Por consiguiente, ahora es el momento para determinar la necesidad de normar las tasas por parte del IASB, así se anticiparía a la futura crisis que se prevé en el mediano plazo de una gran recesión mundial por efecto; además, de la caída del comercio internacional, que generaría el hundimiento de las tasas de intereses a valores negativos.

2. Se ha demostrado que la única manera de evitar el abuso del deterioro de activos tangibles e intangibles, es propiciando tasas bajas de interés. $\mathrm{Y}$ su tendencia internacional futura, es hacia la baja. Si se fijan parámetros a nivel global, los Contadores Públicos en calidad de Auditores Externos, contribuirían con la obligación de hacer cumplir la norma.

3. El mundo está más que sorprendido con los escándalos internacionales de la corrupción. Y una medida en la dirección correcta hacia la minimización de este flagelo social, es normar las tasas de interés para efectos de la aplicación uniforme y previsible de las normas internacionales de contabilidad. Las economías de los países y la sociedad en su conjunto, se beneficiarán al reducir la oportunidad de abusar del uso del crédito fiscal para efecto del pago de impuestos a la renta, en desmedro de los presupuestos nacionales de los países en desarrollo. 


\section{REFERENCIAS BIBLIOGRÁFICAS}

1. BARBERA, J. GUTIÉRREZ, A. SIABA, J. (2015). Hacia una Normalización Posible. Fundación Konrad Adenauer en Argentina. Documento de Trabajo No. 95. Buenos Aires. Argentina.

2. CRUZ, S. (2000). Determinación del Tipo de descuento a emplear para el cálculo del valor de rendimiento de una empresa. Universidad de Almería. España.

3. FERRER, A. (2013, IV-6)Revisando las NIIF: NIC 36 Deterioro del Valor de los Activos. Revista "Actualidad Empresarial” No. 221. Segunda Quincena de Diciembre 2010. Lima. Perú.

4. FERRER, A. (2013, IV-11.Revisando las NIIF: NIC 36 Deterioro del Valor de los Activos. Revista "Actualidad Empresarial” No. 277. Segunda Quincena de Abril 2013. Lima. Perú.

5. FERRER, A. (2013, IV-13. Revisando las NIIF: NIC 36 Deterioro del Valor de los Activos. Revista "Actualidad Empresarial” No. 277. Segunda Quincena de Abril 2013. Lima. Perú.

6. GALLARDO, E. (2010). Evaluación del Deterioro del Valor de los Activos Fijos en una Empresa dedicada a la Fabricación de Helados. Tesis para Contador Público y Auditor. Universidad de San Carlos de Guatemala.
7. IASB. (2015). Norma Internacional de Contabilidad No. 36. Deterioro de Activos. Londres. Gran Bretaña.

8. (IASCF). (2015). Casos de Normas Internacionales de Contabilidad. Fundación del Comité de Estándares Internacionales de Contabilidad. Londres. Gran Bretaña.

9. KIFF, J. (2012). ¿Qué significa Libor? Revista "Finanzas \& Desarrollo". Fondo Monetario Internacional. New York. U.S.A.

10.MARTÍNEZ, J. (20126). Economía global: menor crecimiento y mayor desigualdad. Diario Gestión No. 7325. 09/09/29016. Lima. Perú.

11.PAREDES, M. (2012).Tasas Internacionales de Interés. Superintendencia de Bancos y Seguros del Ecuador.

12.SUMBAD, Y SIGUAM. (2010). Deterioro y Baja de Activos Fijos de la Compañía de Transportes Arcentales Cía., Ltda.,para el año 2010. Tesina para Contador Público Colegiado. Universidad de Cuenca. Ecuador.

13.ZANABRIA, P. (2009). Entendiendo la libor como benchmark. Revista "Moneda". Página. 49-52. Banco Central de Reserva del Perú. Lima. Perú. 